\title{
The correlation between solar and geomagnetic activity - Part 1: Two-term decomposition of geomagnetic activity
}

\author{
Z. L. Du \\ Key Laboratory of Solar Activity, National Astronomical Observatories, Chinese Academy of Sciences, \\ Beijing 100012, China
}

Received: 30 November 2010 - Revised: 29 May 2011 - Accepted: 17 July 2011 - Published: 5 August 2011

\begin{abstract}
By analyzing the logarithmic relationship between geomagnetic activity as represented by the annual $a a$ index and solar magnetic field activity as represented by the annual sunspot number $\left(R_{\mathrm{Z}}\right)$ during the period 1844-2010, a a is shown to lie in between two lines defined solely by $R_{\mathrm{z}}$. Two ways can be used to decompose the $a$ a index into two components. One is decomposing $a a$ into the sum of the baseline $\left(a a_{\mathrm{b}}\right)$ and the remainder $\left(a a_{\mathrm{u}}\right)$ with a null correlation. Another is dividing the top-line $\left(a a_{\mathrm{t}}\right)$ into the sum of $a a$ and the remainder $\left(a a_{\mathrm{d}}\right)$ with a null correlation. The first decomposition is similar to the traditional one. The second decomposition implies a nonlinear relationship of $a a$ with $R_{\mathrm{z}}\left(a a_{\mathrm{t}}\right)$ and a decay process $\left(a a_{\mathrm{d}}\right)$. Therefore, $a a_{\mathrm{t}}=a a+a a_{\mathrm{d}}=a a_{\mathrm{b}}+a a_{\mathrm{u}}+a a_{\mathrm{d}}$ : (i) $a a_{\mathrm{t}}$ is related to the solar energy potential of generating geomagnetic activity (associated with $R_{\mathrm{Z}}$ ); (ii) $a a_{\mathrm{b}}$ is related to transient phenomena; (iii) $a a_{\mathrm{u}}$ is related to recurrent phenomena; and (iv) $a a_{\mathrm{d}}$ is related to the energy loss in the transmission from solar surface to the magnetosphere and ionosphere that failed to generate geomagnetic activity.
\end{abstract}

Keywords. Magnetospheric physics (Solar windmagnetosphere interactions)

\section{Introduction}

Since Mayaud (1972) designed the geomagnetic activity $a a$ index from the 3-hourly $\mathrm{K}$ indices at two near-antipodal midlatitude stations, numerous authors have used it to analyze the global geomagnetic activity and its correlation with solar activity (Schatten et al., 1978; Feynman, 1982; Legrand and Simon, 1989a; Nevanlinna and Kataja, 1993; Lukianova

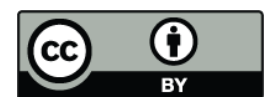

Correspondence to: Z. L. Du

(zldu@nao.cas.cn) et al., 2009; Du et al., 2009; Du, 2011a). The $a a$ index appears an 11-year cycle similar to that of sunspot activity, as described by the Zurich sunspot number $\left(R_{\mathrm{z}}\right)$. Studying the relationship between geomagnetic activity, as represented by $a a$, and solar activity, as represented by $R_{\mathrm{z}}$, may contribute to understanding the origin and formation of the former.

Geomagnetic activities have long been known to be correlated with solar activities (Snyder et al., 1963; Russell and McPherron, 1973; Garrett et al., 1974; Feynman and Crooker, 1978). Geomagnetic activities can be resulted from variable current systems formed in the magnetosphere and ionosphere, such as the ring current and auroral ionospheric current, which are strongly modulated by solar activities via the interaction of the magnetosphere with solar winds (Feynman, 1980; Legrand and Simon, 1989a,b; Demetrescu and Dobrica, 2008) or others (Legrand and Simon, 1981, 1989a; Stamper et al., 1999; Tsurutani et al., 2006). It is believed that the geomagnetic activity is well associated with the solar wind speed $(V)$, the southward component $\left(B_{\mathrm{Z}}\right)$ of the interplanetary magnetic field (IMF) and their product (Snyder et al., 1963; Russell and McPherron, 1973; Garrett et al., 1974; Crooker et al., 1977; Svalgaard, 1977; Feynman, 1980; Wang and Sheeley, 2009). In general, the magnetosphere exhibits approximately a linear response to the solar wind drivers. However, a nonlinear behavior is significant in the declining phase of a solar cycle, which is related to increased solar wind speeds (Legrand and Simon, 1989a,b; Venkatesan et al., 1991; Echer et al., 2004; Johnson and Wing, 2005).

The geomagnetic activity results from two main solar sources (Legrand and Simon, 1981; Venkatesan et al., 1982; Feynman, 1982; Legrand and Simon, 1989a,b; Gonzalez et al., 1990; Venkatesan et al., 1991; Echer et al., 2004). The first source is related to transient phenomena such as solar flares, prominence eruptions, and coronal mass ejections (CMEs), and follows the sunspot cycle (Venkatesan et al., 1991). The second source is related to recurrent phenomena (high-speed solar wind streams) and tends to peak in

Published by Copernicus Publications on behalf of the European Geosciences Union. 


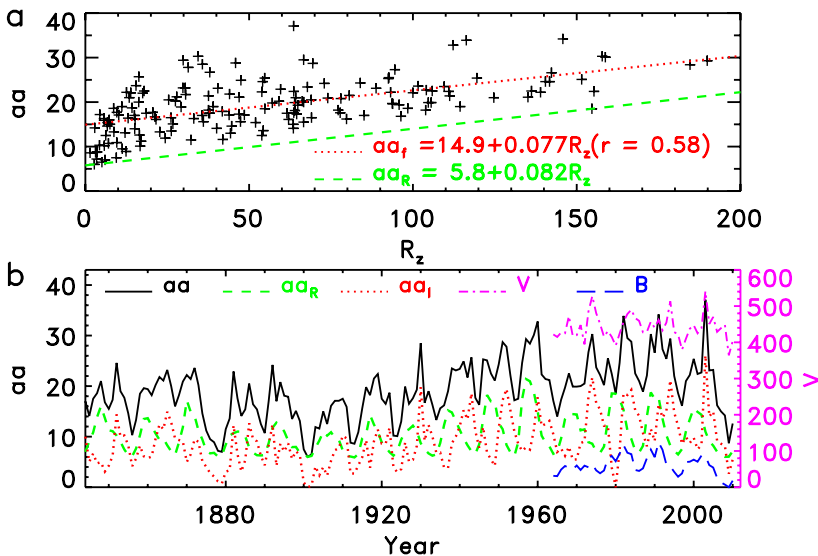

Fig. 1. (a) Scatter plot of $a a$ against $R_{\mathrm{Z}}$ (pluses), linear fit (dotted), and baseline $a a_{\mathrm{R}}$ (dashed). (b) The time series of $a a$ (solid), $a a_{\mathrm{R}}$ (dashed) from Eq. (2) and $a a_{\mathrm{I}}$ (dotted) from Eq. (3) since 1844. The time series of $V$ (dash-dotted) and $B$ (long-dashed) since 1964 are also shown for comparison, with the $B$ values so scaled that they can be clearly seen.

the declining phase or at the solar minimum of the cycle (Legrand and Simon, 1981; Venkatesan et al., 1982; Legrand and Simon, 1989a,b; Tsurutani et al., 2006). Feynman (1982) analyzed the relationship between the annual $a a$ and $R_{\mathrm{Z}}$ series from 1869 to 1975 and found that the $a a$ values are all above a base line $\left(a a_{\mathrm{R}}\right)$ that is linearly related to $R_{\mathrm{z}}$. Then, she decomposed the $a a$ index into two equally strong periodic components: $a a_{\mathrm{R}}$ and the remainder $a a_{\mathrm{I}}=a a-a a_{\mathrm{R}}$. The first one (the "short lived" $\mathrm{R}$ component) is associated with the transient phenomena and follows the sunspot cycle, while the second one (the "slowly varying" interplanetary I component) is associated with the recurrent phenomena and is almost $180^{\circ}$ out of phase with the sunspot cycle (Hathaway and Wilson, 2006). Legrand and Simon (1989a) classified the geomagnetic activity in four classes: the magnetic quiet activity, the recurrent activity, the fluctuating activity, and the shock activity. At mid-latitude, the geomagnetic activity is sensitive both to the auroral phenomena (particle precipitations, substorms, and auroras) which are at the origin of the auroral electrojet (AE) activity, and to the equatorial ring current which is the source of the geomagnetic storms (Legrand and Simon, 1989a,b). Therefore, the $a a$ index is an integral effect of various sources of geomagnetic activity.

The relationship between the solar and geomagnetic activity is not a simple linear one (Feynman, 1983; Legrand and Simon, 1983) due to various sources of geomagnetic activity. Decomposing the geomagnetic activity ( $a a$ index) into different parts may help understand its origins.

In this paper, we first reexamine the work of Feynman (1982) to decompose the annual $a a$ index for the data available (Sect. 2) into two components based on a linear relationship between $a a$ and $R_{\mathrm{Z}}$ (Sect. 3). To elucidate how $a a$ depends on $R_{\mathrm{z}}$, we analyze the scatter plot of $\ln a a$ against $\ln R_{\mathrm{Z}}$ in Sect. 4. All the data points are found to be in between the two lines of baseline $\left(\ln a a_{\mathrm{b}}\right)$ and top-line $\left(\ln a a_{\mathrm{t}}\right)$. According to the baseline, the $a a$ index can be decomposed into two components: $a a_{\mathrm{b}}$ and the remainder $a a_{\mathrm{u}}=a a-a a_{\mathrm{b}}$ with a null correlation (Sect. 4.1). This decomposition is similar to that of Feynman (1982), but the relationship between $a a_{\mathrm{b}}$ and $R_{\mathrm{Z}}$ is nonlinear. The top-line provides another way to decompose $a a_{\mathrm{t}}$ into two components: $a a$ and $a a_{\mathrm{d}}=a a_{\mathrm{t}}-a a$ with a null correlation (Sect. 4.2). We attempt to explain the second decomposition by a nonlinear model (Sect. 4.2.1) and a decay process (Sect. 4.2.2). The $a a$ index is the remainder of $a a_{\mathrm{t}}$ after decay $a a_{\mathrm{d}}$. Some conclusions are discussed and summarized in Sect. 5.

\section{Data}

To suppress the high frequencies involved in the solar rotation and seasonal variations, in this study the geomagnetic activity is represented by the annual averages of $a a$ index (Mayaud, 1972) using reliable values since $1868^{1}$ and the equivalent ones from measurements in Finland from 1844 to 1867 (Nevanlinna and Kataja, 1993). The solar (magnetic field) activity is represented by the annual averages of $R_{\mathrm{z}}^{2}$ from 1844 to 2010. In addition, for comparison we use the annual speed $(V)$ and magnetic field $(B)$ of solar wind from the OMNI data sets since $1964^{3}$, and the Dst (disturbance storm time) ${ }^{4}$ index representing the ring current (Gonzalez et al., 1990) since 1958.

\section{Two-term decomposition of $a a$ according to the linear relationship between $a a$ and $R_{\mathrm{z}}$}

In this section, we repeat the Feynman (1982) work to decompose $a a$ into two components, using the data from 1844 to 2010. Figure 1a shows the scatter plot of $a a$ against $R_{\mathrm{Z}}$ (pluses). The dotted line indicates the linear fit of $a a$ to $R_{\mathrm{Z}}$, with the regression equation given by

$a a=14.9+0.077 R_{\mathrm{Z}}$.

It is seen that the data points are all above the (dashed) baseline, defined by the lower envelope,

$a a_{\mathrm{R}}=5.8+0.082 R_{\mathrm{Z}}$.

The remainder of $a a$ is then

$a a_{\mathrm{I}}=a a-a a_{\mathrm{R}}$.

Figure $1 \mathrm{~b}$ shows the time series of $a a$ (solid), $a a_{\mathrm{R}}$ (dashed), and $a a_{\mathrm{I}}$ (dotted) since 1844. The correlation coefficient between the two components, $a a_{\mathrm{R}}$ and $a a_{\mathrm{I}}$, is almost zero

\footnotetext{
${ }^{1} \mathrm{ftp} / / / \mathrm{ftp} . n g d c$. noaa.gov/STP/SOLAR_DATA/RELATED_ INDICES/AA_INDEX/

${ }^{2} \mathrm{http}: / /$ www.ngdc.noaa.gov/stp/spaceweather.html

$3 \mathrm{ftp}: / /$ nssdcftp.gsfc.nasa.gov/spacecraft_data/omni/

${ }^{4}$ http://www.ngdc.noaa.gov/stp/spaceweather.html
} 
Table 1. Correlation coefficients $(r)$ between $a a, R_{\mathrm{Z}}, a a_{\mathrm{R}}, a a_{\mathrm{I}}, V$, and $B$.

\begin{tabular}{lccccc}
\hline$r$ & $a a$ & $a a_{\mathrm{R}}\left(R_{\mathrm{Z}}\right)$ & $a a_{\mathrm{I}}$ & $V$ & $B$ \\
\hline$a a$ & 1 & 0.58 & 0.79 & 0.74 & 0.83 \\
$a a_{\mathrm{R}}\left(R_{\mathrm{Z}}\right)$ & & 1 & -0.05 & -0.06 & 0.78 \\
$a a_{\mathrm{I}}$ & & & 1 & 0.84 & 0.31 \\
$V$ & & & & 1 & 0.32 \\
\hline
\end{tabular}

( $r=-0.05)$, implying that $a a_{\mathrm{I}}$ has a $90^{\circ}(\sim 3-\mathrm{yr})$ rather than a $180^{\circ}$ (Feynman, 1982; Li, 1997; Hathaway and Wilson, 2006) phase shift to $a a_{\mathrm{R}}$. The phase shift is related to the time delay of $a a$ to $R_{\mathrm{Z}}$ (Echer et al., 2004; Du, 2011b). Figure 1 b also shows $V$ (dash-dotted) and $B$ (long-dashed) since 1964 for comparison. The correlation coefficients between these parameters are listed in Table 1.

One sees in Table 1 that: (i) $a a$ is positively correlated with $R_{\mathrm{Z}}(r=0.58), a a_{\mathrm{R}}(r=0.58), a a_{\mathrm{I}}(r=0.79), V(r=0.74)$, and $B(r=0.83)$; (ii) $a a_{\mathrm{R}}\left(R_{\mathrm{Z}}\right)$ is well correlated with $B$ ( $r=0.78)$ but has a null correlation with $V(r=-0.06)$; and (iii) $a a_{\mathrm{I}}$ has a much higher correlation with $V(r=0.84)$ than with $B(r=0.31)$. Therefore, $a a_{\mathrm{R}}$ and $a a_{\mathrm{I}}$ are well associated with $B$ and $V$, respectively.

The two components of $a a_{\mathrm{R}}$ and $a a_{\mathrm{I}}$ have been explained by two main solar sources of geomagnetic activity (Legrand and Simon, 1981; Venkatesan et al., 1982; Legrand and Simon, 1989a; Gonzalez et al., 1990; Venkatesan et al., 1991; Legrand and Simon, 1989a,b; Echer et al., 2004): (i) $a a_{\mathrm{R}}$ is related to solar flares and coronal mass ejections (CMEs); and (ii) $a a_{\mathrm{I}}$ is related to high-speed solar wind streams and is out of phase with the sunspot cycle (Feynman, 1982).

\section{Two-term decomposition of a a according to the loga- rithmic relationship between $a a$ and $R_{\mathrm{z}}$}

To elucidate how $a a$ depends on $R_{\mathrm{z}}$, we analyze the correlation between the natural logarithms of the annual $a a$ and $R_{\mathrm{Z}}$ from 1844 to 2010 . The scatter plot of $\ln$ aa against $\ln R_{\mathrm{Z}}$ is shown in Fig. 2. The dotted line indicates the linear fit of $\ln a a$ to $\ln R_{\mathrm{z}}$, with the regression equation given by

$\ln a a=2.13+0.21 \ln R_{\mathrm{z}}, \quad$ or $\quad a a=8.41 R_{\mathrm{z}}^{0.21}$.

The positive correlation between $\ln a a$ and $\ln R_{\mathrm{Z}}(r=0.67)$ is stronger than that between $a a$ and $R_{\mathrm{Z}}(r=0.58)$, implying that $a a$ varies preferably nonlinearly with $R_{\mathrm{z}}$. Also shown in the figure are the years of the maximum (triangles) and minimum (circles) amplitudes of the sunspot cycle.

One very prominent property in Fig. 2 is that the data points are all in between the two parallel (dashed) lines with

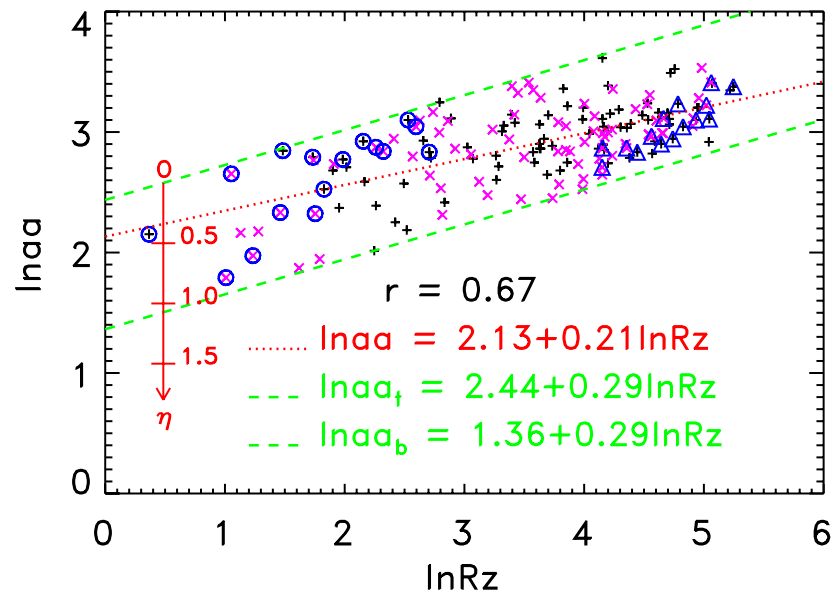

Fig. 2. Scatter plot of $\ln a a$ against $\ln R_{\mathrm{Z}}$ (black pluses for oddnumbered cycles and purple crosses for even-numbered cycles) with a correlation coefficient of $r=0.67$. The dotted line indicates the linear fit of $\ln a a$ to $\ln R_{\mathrm{Z}}$. The data points are all in between the two parallel (dashed) lines of $\ln a a_{\mathrm{t}}$ (top) and $\ln a a_{\mathrm{b}}$ (base). Triangles and circles denote the years of the maximum and minimum amplitudes of the sunspot cycle, respectively. The coordinate axis $\eta$ describes decay strength.

their equations defined (from two points on baseline or topline) by

$\ln a a_{\mathrm{b}}=1.36+0.29 \ln R_{\mathrm{z}}, \quad$ or $\quad a a_{\mathrm{b}}=e^{1.36} R_{\mathrm{z}}^{2 / 7}$

and

$\ln a a_{\mathrm{t}}=2.44+0.29 \ln R_{\mathrm{Z}}, \quad$ or $\quad a a_{\mathrm{t}}=e^{2.44} R_{\mathrm{Z}}^{2 / 7}$.

The above equations suggest that a certain level of solar activity $\left(R_{\mathrm{z}}\right)$ is associated with at least (most) $a a_{\mathrm{b}}\left(a a_{\mathrm{t}}\right)$ of the geomagnetic activity. From $a a_{\mathrm{b}}$ and $a a_{\mathrm{t}}$, two new indices, $a a_{\mathrm{u}}$ and $a a_{\mathrm{d}}$, can be defined such that

$a a=a a_{\mathrm{b}}+a a_{\mathrm{u}}, \quad$ or $\quad a a_{\mathrm{u}}=a a-a a_{\mathrm{b}}$

and

$a a_{\mathrm{t}}=a a+a a_{\mathrm{d}}, \quad$ or $\quad a a_{\mathrm{d}}=a a_{\mathrm{t}}-a a$.

The null correlation of $a a_{\mathrm{b}}$ with $a a_{\mathrm{u}}(r=0.09)$ implies that $a a$ can be decomposed into two independent components: $a a_{\mathrm{b}}$ and $a a_{\mathrm{u}}$. Similarly, the null correlation of $a a$ with $a a_{\mathrm{d}}$ ( $r=-0.0003)$ implies that $a a_{\mathrm{t}}$ can be divided into two independent orthogonal terms: $a a$ and $a a_{\mathrm{d}}$.

As $a a_{\mathrm{b}}$ and $a a_{\mathrm{t}}$ are strictly defined by Eqs. (5)-(6), the correlation coefficient of $\ln a a_{\mathrm{b}}$ or $\ln a a_{\mathrm{t}}$ with $\ln R_{\mathrm{z}}$ is one, having the same periodicity, phase, and relative amplitude, so that $a a_{\mathrm{b}}\left(a a_{\mathrm{t}}\right)$ follows $R_{\mathrm{z}}(r=0.94)$ well. If $a a$ were proportional to $a a_{\mathrm{b}}\left(a a_{\mathrm{t}}\right)$, the remainder $a a_{\mathrm{u}}\left(a a_{\mathrm{d}}\right)$ would be random. However, the correlation of $a a_{\mathrm{u}}$ with $a a(r=0.84)$ is stronger than that of $a a_{\mathrm{b}}$ with $a a(r=0.62)$, and the correlation of $a a_{\mathrm{d}}$ with $a a_{\mathrm{t}}(r=0.78)$ is stronger than that of $a a$ 


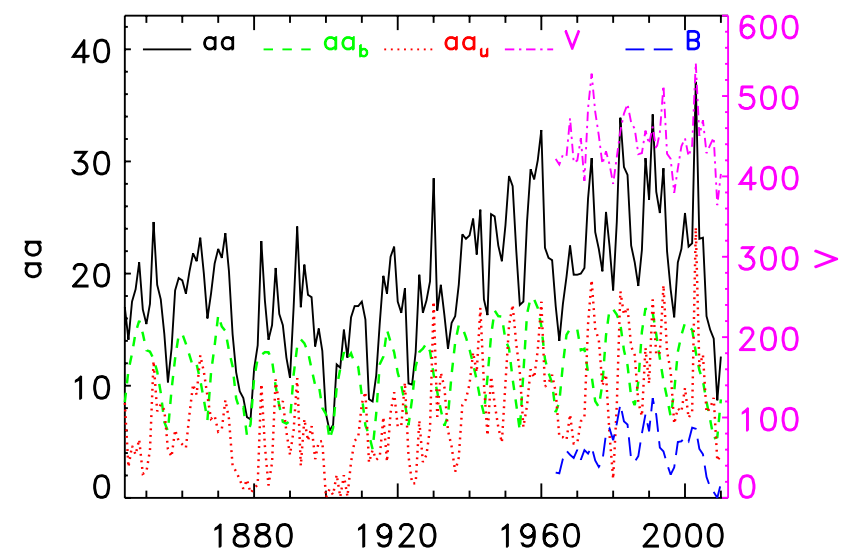

Fig. 3. Time series of $a a$ (solid), $a a_{\mathrm{b}}$ (dashed) from Eq. (5), $a a_{\mathrm{u}}$ (dotted) from Eq. (7), $V$ (dash-dotted), and $B$ (long-dashed), with the $B$ values so scaled that can be clearly seen.

with $a a_{\mathrm{t}}(r=0.62)$, Therefore, some underlying information must exist in both $a a_{\mathrm{u}}$ and $a a_{\mathrm{d}}$.

The strong correlation of $a a_{\mathrm{d}}$ with $R_{\mathrm{z}}(r=0.75)$ and the null correlation of $a a_{\mathrm{d}}$ with $a a(r=0.00)$ imply that $a a_{\mathrm{d}}$ is well associated with the level of $R_{\mathrm{Z}}$ (solar activity). In contrast, the strong correlation of $a a_{\mathrm{u}}$ with $a a(r=0.84)$ and the null correlation of $a a_{\mathrm{u}}$ with $R_{\mathrm{Z}}(r=0.07)$ imply that $a a_{\mathrm{u}}$ reflects the variation in $a a$ (geomagnetic activity).

\subsection{Two-term decomposition of $a a$ based on the baseline}

Similar to Sect. 3, one decomposition method of the $a a$ index is according to the baseline $\left(a a_{\mathrm{b}}\right)$, as the $a a$ values are all above $a a_{\mathrm{b}}$ (Fig. 2). Figure 3 shows the two components, $a a_{\mathrm{b}}$ (dashed) from Eq. (5) and $a a_{\mathrm{u}}$ (dotted) from Eq. (7), together with $a a$ (solid), $V$ (dash-dotted), and $B$ (long-dashed) for comparison. The correlation coefficients between these parameters are listed in Table 2.

The following may by noted in Table 2: (i) $a a$ is positively correlated with $a a_{\mathrm{b}}, a a_{\mathrm{u}}, V$, and $B$; (ii) $a a_{\mathrm{b}}$ is well correlated with $B(r=0.83)$ but has a null correlation with $V(r=0.03)$; and (iii) $a a_{\mathrm{u}}$ has a much higher correlation with $V(r=0.85)$ than with $B(r=0.41)$. Therefore, $a a_{\mathrm{b}}$ and $a a_{\mathrm{u}}$ are well associated with $B$ and $V$, respectively, reflecting the two sources of geomagnetic activity.

This decomposition is similar to that in Sect. 3. The $a a_{\mathrm{b}}$ and $a a_{\mathrm{u}}$ terms in this section correspond similarly to the $\mathrm{R}$ and I components in the previous section, respectively. The main discrepancy is that the $\mathrm{R}$ component is linearly related to $R_{\mathrm{Z}}$ (Eq. 2), while $a a_{\mathrm{b}}$ is related to $R_{\mathrm{Z}}$ in the form of a power-law (Eq. 5). Thus, the $a a_{\mathrm{b}}$ component can be explained to be nonlinearly related to solar flares and CMEs, and the $a a_{\mathrm{u}}$ component is related to high-speed solar wind streams having a $90^{\circ}(\sim 3-\mathrm{yr})$ phase shift to $a a_{\mathrm{b}}$.

It should be pointed out in Fig. 2 that there is a maximum $\left(a a_{\mathrm{t}}\right)$ for the $a a$ values and that there is a maximum
Table 2. Correlation coefficients between $a a, a a_{\mathrm{b}}, a a_{\mathrm{u}}, V$ and $B$.

\begin{tabular}{lccccc}
\hline$r$ & $a a$ & $a a_{\mathrm{b}}$ & $a a_{\mathrm{u}}$ & $V$ & $B$ \\
\hline$a a$ & 1 & 0.62 & 0.84 & 0.74 & 0.83 \\
$a a_{\mathrm{b}}$ & & 1 & 0.10 & 0.03 & 0.83 \\
$a a_{\mathrm{u}}$ & & & 1 & 0.85 & 0.41 \\
\hline
\end{tabular}

$\left(a a_{\mathrm{t}}-a a_{\mathrm{b}}\right)$ for the $a a_{\mathrm{u}}$ component, which is part of $a a$ above the baseline $a a_{\mathrm{b}}$ (Eq. 7). This result suggests that $R_{\mathrm{Z}}$ is associated with a certain amount of solar energy $\left(E_{\mathrm{t}}\right)$ potential of generating geomagnetic activity $(a a)$ whose maximum $\left(a a_{\mathrm{t}}\right)$ is related to $E_{\mathrm{t}}$. The energy $\left(E_{\mathrm{t}}\right)$ consists of three parts: (i) the first part $\left(E_{\mathrm{b}}\right)$, being related to the transient phenomena, generates the geomagnetic activity $\left(a a_{\mathrm{b}}\right)$ that is well associated with $R_{\mathrm{z}}\left(a a_{\mathrm{b}} \propto R_{\mathrm{z}}^{2 / 7}\right)$; (ii) the second part $\left(E_{\mathrm{u}}\right)$, being related to the recurrent phenomena, generates the geomagnetic activity $\left(a a_{\mathrm{u}}\right)$ that is almost uncorrelated with the former $\left(a a_{\mathrm{b}}\right)$; and (iii) the third part $\left(E_{\mathrm{d}}=E_{\mathrm{t}}-E_{\mathrm{b}}-E_{\mathrm{u}}\right)$, generating no geomagnetic activity, might be interpreted as the energy loss in the energy transmission from solar surface to the magnetosphere and ionosphere, which may involve very complex processes (see also Sect. 4.2 and Discussion) and may be related to the time delay of geomagnetic activity $(a a)$ to solar activity $\left(R_{\mathrm{z}}\right)$.

For the decomposition in Fig. 2, the $a a$ values are all above the baseline $a a_{\mathrm{R}}$ (Eq. 2), while there seems to be no upper limit for the $a a$ or $a a_{\mathrm{I}}$ (Eq. 3) values shown in Fig. 1a - at least such a limit (if existing) is not apparent.

\subsection{Two-term decomposition of $a a$ based on the top-line}

Another alternate decomposition method is by use of the topline $\left(a a_{\mathrm{t}}\right)$, as the $a a$ values are all below $a a_{\mathrm{t}}$ (Fig. 2). The two components, $a a$ and $a a_{\mathrm{d}}$ from Eqs. (6) and (8), have a null correlation $(r \sim 0)$, implying that the $a a$ index has a $90^{\circ}$ ( $\sim 3$-yr) phase shift to $a a_{\mathrm{d}}$. This decomposition might be explained by a nonlinear model and a decay process.

\subsubsection{A nonlinear model}

Suppose that the variation rate of $a a$ is proportional to that of $R_{\mathrm{Z}}$,

$\frac{\Delta a a}{a a} \propto \frac{\Delta R_{\mathrm{z}}}{R_{\mathrm{z}}}, \quad$ or $\quad \frac{\partial a a}{\partial R_{\mathrm{z}}}=\gamma \frac{a a}{R_{\mathrm{z}}}$,

where $\gamma$ is called "response efficiency" of $a a$ to $R_{\mathrm{z}}$. The solution to this equation is in the form of

$\ln a a=\beta+\gamma \ln R_{\mathrm{Z}}, \quad$ or $\quad a a\left(R_{\mathrm{Z}}\right)=e^{\beta} R_{\mathrm{Z}}^{\gamma}$, 
where $\beta$ is an integral constant. Thus, Eq. (10) can be taken as a general form of Eqs. (5)-(6) for

$$
\begin{gathered}
\gamma=2 / 7, \\
\beta_{\mathrm{t}}=2.44, \\
\beta_{\mathrm{b}}=1.36 .
\end{gathered}
$$

It suggests from Eqs. (10) and (4) that aa varies with $R_{\mathrm{Z}}$ nonlinearly rather than linearly. In fact, the values of $\gamma$ for the top- and base-line are not strictly equal to one another. By carefully examining the top-line in Fig. 2, two possible values of $\gamma$ are $\gamma_{\mathrm{t}}=0.27$ and 0.31 (from two different pairs of the upper envelope), corresponding to $\beta_{\mathrm{t}}=2.49$ and 2.39, respectively. We have taken an average of $\gamma_{\mathrm{t}}=0.29$, which is equal to $\gamma_{\mathrm{b}}=0.29$ for the baseline, and $\beta_{\mathrm{t}}=2.44$ correspondingly. Therefore, $\gamma$ and $\beta$ have uncertainties of about $\Delta \gamma=0.02$ and $\Delta \beta=0.05$, respectively. The $\gamma$ value reflects the generation efficiency of $a a$ by solar (activity) energy that is related to $R_{\mathrm{z}}$, only about $2 / 7$ (29\%) of the relative variation in $R_{\mathrm{z}}$ being related to the variation in $a a$ in terms of annual averages.

\subsubsection{A decay process}

From Eqs. (5), 6), and (11) we have

$a a_{\mathrm{b}}=e^{-\left(\beta_{\mathrm{t}}-\beta_{\mathrm{b}}\right)} a a_{\mathrm{t}}=e^{-1.08} a a_{\mathrm{t}}$.

In accordance with this equation, all the data points in Fig. 2 can be divided into many groups, each lying on an oblique narrow stripe (belt) parallel to the top-line. These stripes are denoted by a coordinate axis $(\eta)$.

Suppose that $a a$ undergoes a decay process in terms of the variable $z$,

$-\Delta a a \propto a a \Delta z, \quad$ or $\quad \frac{\partial a a}{\partial z}=-\frac{1}{L} a a$,

where $L$ is the decay scale. Its solution is

$a a(z)=e^{-z / L} a a_{0}$,

where $a a_{0}$ is an integral constant. Because the $a a$ values are all in between the two lines (Fig. 2), $z=0$ can be taken as the top-line and $a a_{0}=a a_{\mathrm{t}}$ as the boundary condition, so that

$a a(z)= \begin{cases}e^{-z / L} a a_{\mathrm{t}}, & 0 \leq z \leq 1.08 L, \\ a a_{\mathrm{b}}, & z>1.08 L,\end{cases}$

and the decay term is

$$
\begin{aligned}
a a_{\mathrm{d}}(z) & =a a_{\mathrm{t}}(z)-a a(z) \\
& = \begin{cases}\left(1-e^{-z / L}\right) a a_{\mathrm{t}}, & 0 \leq z \leq 1.08 L \\
a a_{\mathrm{t}}-a a_{\mathrm{b}}, & z>1.08 L\end{cases}
\end{aligned}
$$

The above two equations can be easily explained if $z$ is temporarily taken as a length variable with its origin at the "outer surface" of the magnetosphere and its positive direction towards the Earth. (i) At first, the interaction of solar activities (solar winds and CMEs, etc.) with the magnetosphere produces geomagnetic activity $\left(a a=a a_{\mathrm{t}}\right)$, which is the top-line in Fig. 2. Then $a a_{\mathrm{t}}$ undergoes a decay process according to $a a_{\mathrm{d}}(z)=\left(1-e^{-z / L}\right) a a_{\mathrm{t}}$ for $0 \leq z \leq 1.08 L$ as it attempts to go through the decay range (ionosphere as well as magnetosphere, hereafter DR, where $a a$ decays). Only the remainder $a a(z)=a a_{\mathrm{t}}-a a_{\mathrm{d}}(z)=e^{-z / L} a a_{\mathrm{t}}$ is observed after the DR. Various decays, due to various thicknesses and densities of the DR for different districts or different time periods (and different solar activities), constitute the randomly scattered points of $a a$ in Fig. 2 (the random distribution of $a a_{\mathrm{d}}$ or $a a$ is the cause of the null correlation between them). (ii) For $z>1.08 L$, a $a$ has already passed over the DR and will not decay further. As the maximum decay in the whole DR is $\overline{a a}_{\mathrm{d}}=a a_{\mathrm{t}}-a a_{\mathrm{b}}$, the minimum $a a$ is then $\underline{a a}=a a_{\mathrm{t}}-\overline{a a}_{\mathrm{d}}=a a_{\mathrm{b}}$, which is the baseline in Fig. 2 .

It should be noted that the decay $a a_{\mathrm{d}}$ is well correlated with $a a_{\mathrm{t}}(r=0.78)$ or $R_{\mathrm{Z}}(r=0.75)$, meaning that a higher level of $R_{\mathrm{z}}$ tends to be associated with a larger $a a_{\mathrm{d}}$ or $z$. Although a stronger (weaker) solar activity $R_{\mathrm{Z}}$ (corresponding to the energy $E_{\mathrm{t}}$ ) is related to a higher (lower) level of $a a_{\mathrm{t}}$, more (less) decays (or the "energy loss" $E_{\mathrm{d}}$ ) will also be produced while transmitting through the DR. When $a a$ has finally passed over the DR, only a part is left: the observed $a a=a a_{\mathrm{t}}-a a_{\mathrm{d}}$ (or the energy $E=E_{\mathrm{t}}-E_{\mathrm{d}}$ ). Thus, the variable $z$ is in fact a quantity to describe the decay strength (or the energy loss $E_{\mathrm{d}}$ ) in the DR, and $L$ is the decay scale (or the maximum energy loss $E_{\mathrm{d}, \max }$ ). The $z / L$ value reflects the energy loss rate $\left(E_{\mathrm{d}} / E_{\mathrm{d} \text {, max }}\right)$, which is related to the intensity and orientation of the solar dipole (Simon and Legrand, 1989), the topology and density (especially the ion density) of the DR, the size and shape of the current sheet, or the ion inertial scale (Leamon et al., 2000; Matthaeus et al., 2008). The formation is related to the interaction mechanism of solar activities (solar winds and CMEs, etc.) with the magnetosphere (and of the fast with slow solar winds) and the state (strength, velocity, and direction) of the solar wind. More geomagnetic activities can be produced if the solar wind reaches the magnetosphere perpendicularly than in any other direction.

This explanation is, of course, a simplified characterization. In fact, the geomagnetic activity may be produced and decayed all the way from the magnetosphere to the Earth. Magnetic fields play a determining role in the formation and dynamics of solar activities. Besides sunspots $\left(R_{\mathrm{Z}}\right)$, the magnetic fields also produce other phenomena of solar activities, such as solar flares, prominence eruptions, energetic protons, CMEs, and solar winds (Legrand and Simon, 1989a), in nonlinear processes more or less similar to Eq. (15). As the source activities of geomagnetic activity, these activities have already undergone decays before arriving at the magnetosphere (e.g., in solar corona), and play a role of midprocesses from the solar magnetic field activity to geomagnetic activity (see Discussions). From the perspective of the overall result, the formation of $a a$ can be totally expressed 


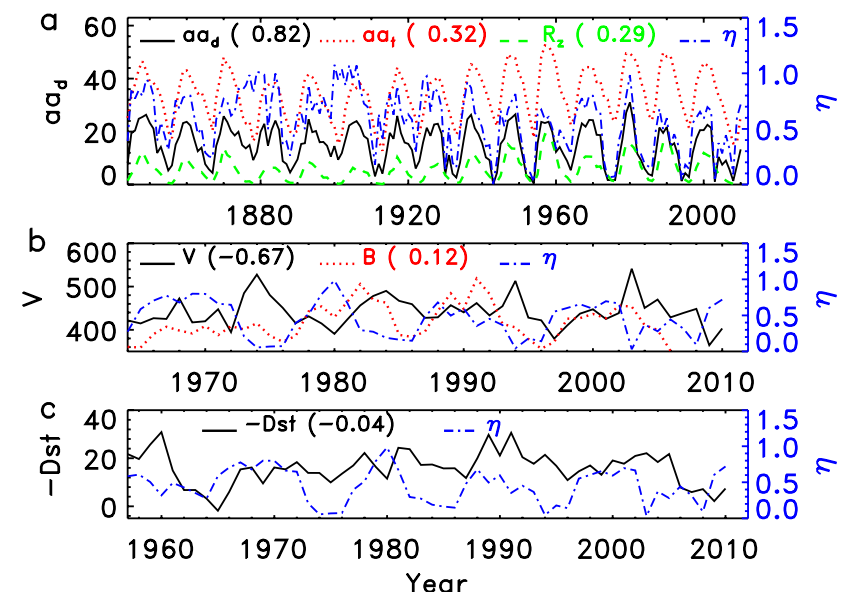

Fig. 4. (a) $a a_{\mathrm{d}}$ (solid) from Eq. (8), $a a_{\mathrm{t}}$ (dotted) from Eq. (6) and $R_{\mathrm{Z}}$ (dashed) since 1844 . (b) $V$ (solid), $B$ (dotted), and $\eta$ (dashdotted) since 1964. (c) -Dst (solid) and $\eta$ (dash-dotted) since 1958. The values of $R_{\mathrm{Z}}$ and $B$ are so scaled that they can be clearly seen. The numbers in brackets indicate the correlation coefficients of the parameters with $\eta$.

as $a a_{\mathrm{t}}$ in Eq. (6), and the decay process can be described by Eq. (16).

\subsection{3 aa's expression and decay index $\eta$}

Combining Eq. (6) with Eq. (15), all the $a a$ values can be mathematically expressed as

$a a=e^{2.44-\eta} R_{\mathrm{Z}}^{2 / 7} \quad(0 \leq \eta \leq 1.08)$,

where

$\eta=z / L$

is called "decay index": the quantity of $z$ normalized to $L$ (Fig. 2), which is related to the decay rate, $a a_{\mathrm{d}} / a a_{\mathrm{t}}=1-$ $e^{-\eta}$. Implied in Eq. (17) is that the geomagnetic activity is generated via a nonlinear relationship with $R_{\mathrm{z}}$ as $R_{\mathrm{z}}^{2 / 7}$ and a decay process according to $e^{-\eta}$.

To study the property of $\eta$, its value can be calculated by Eq. (17),

$\eta=2.44+(2 / 7) \ln R_{\mathrm{Z}}-\ln a a$.

Figure $4 \mathrm{a}$ shows the time series of $a a_{\mathrm{d}}$ (solid), $a a_{\mathrm{t}}$ (dotted), $R_{\mathrm{Z}}$ (dashed), and $\eta$ (dash-dotted) for comparison.

It is apparent in Fig. 4a that a larger $\eta$ corresponds to more decays $\left(a a_{\mathrm{d}}\right)$ and almost, but not quite, a higher $R_{\mathrm{z}}$. In contrast, a smaller $\eta$ (solid) corresponds to less decays and almost, but not quite, a lower $R_{\mathrm{z}}$. The $\eta$ values at the years of solar minima are much more scattered (circles in Fig. 2), ranging from 0.02 to 0.94 with an average of $\bar{\eta}_{\min }=0.36$, while those at the years of solar maxima (triangles) are more concentrated, ranging from 0.50 to 0.94 with an average of $\bar{\eta}_{\max }=0.76$. This may be the reason why the dynamics of
Table 3. Correlation coefficients between $a a, a a_{\mathrm{t}}, a a_{\mathrm{d}}, \eta, V, B$, and Dst.

\begin{tabular}{llllrrrr}
\hline$r$ & $a a$ & $a a_{\mathrm{t}}$ & $a a_{\mathrm{d}}$ & $\eta$ & $V$ & $B$ & Dst \\
\hline$R_{\mathrm{Z}}$ & 0.58 & 0.95 & 0.75 & 0.29 & -0.06 & 0.78 & -0.64 \\
$a a$ & 1 & 0.62 & 0.00 & -0.52 & 0.74 & 0.83 & -0.80 \\
$a a_{\mathrm{t}}$ & & 1 & 0.78 & 0.32 & 0.03 & 0.83 & -0.68 \\
$a a_{\mathrm{d}}$ & & & 1 & 0.82 & -0.48 & 0.41 & -0.27 \\
$\eta$ & & & & 1 & -0.67 & 0.12 & 0.04 \\
$V$ & & & & & 1 & 0.32 & -0.37 \\
$B$ & & & & & & 1 & 0.16 \\
\hline
\end{tabular}

the magnetosphere tends to be more linear at maximum than at minimum (Johnson and Wing, 2005). The larger values of $\eta$ at solar maxima than at solar minima $\left(\bar{\eta}_{\max }>\bar{\eta}_{\text {min }}\right)$ can explain the following phenomenon. The $a a$ index at a higher $R_{\mathrm{z}}$ (around the maximum) tends to undergo more decays and for a longer time when going through the DR, leading to a longer lag time of $a a$ to $R_{\mathrm{Z}}$ at a maximum rather than at a minimum (Wang and Sheeley, 2009; Wilson, 1990; Du, 2011b,c).

In Fig. 2, the $\ln a a-\ln R_{\mathrm{z}}$ data pairs are divided into two parts: one is related to odd-numbered cycles (pluses) and another is related to even-numbered cycles (crosses). The average decay index $\eta(0.57)$ for odd-numbered cycles is slightly less than that $(0.59)$ for even-numbered cycles, which may be related to the stronger correlation for odd-numbered cycles than for even-numbered cycles (Du, 2011b,c).

\subsubsection{The correlations of $\eta$ with $V, B$, and Dst}

Figure $4 \mathrm{~b}$ shows the time series of $V$ (solid), $B$ (dotted), and $\eta$ (dash-dotted) for comparison. One can see that $\eta$ is negatively correlated with $V(r=-0.67)$ and almost independent of $B(r=0.12)$, implying that solar winds with lower speeds tend to decay more. This means that one usually analyzes the correlation between geomagnetic activity and the solar wind speed above a certain value.

Figure $4 \mathrm{c}$ shows the Dst index (solid) and $\eta$ (dash-dotted) for comparison. It is seen that $\eta$ is almost independent of Dst $(r=0.04)$. One possible reason is that Dst is well anticorrelated with $a a(r=-0.8)$, while $a a$ has a null correlation with $a a_{\mathrm{d}}(r=0.00)$. The correlation coefficients involved in these parameters are summarized in Table 3.

It should be pointed out in Table 3 that $\eta$ is well correlated with $a a_{\mathrm{d}}(r=0.82)$, negatively correlated with $V$ $(r=-0.67)$ or $a a(r=-0.52)$, weakly correlated with $R_{\mathrm{Z}}$ or $a a_{\mathrm{t}}(r \sim 0.3)$, and almost uncorrelated with $B$ or Dst $(r \sim 0)$. Therefore, the decay index $(\eta)$ is mainly related to the solar wind speed $(V)$. The reason may be due to that: (i) the (average) speed of solar wind is slower than that of the transient phenomena (e.g., CMEs); (ii) only part of the solar wind energy is transmitted to geomagnetic activity in the interaction with the magnetosphere, which spends time; and (iii) the 
slower solar wind plasma is more difficult to transmit the magnetosphere and decays more than the faster one.

\section{Discussions and conclusions}

By analyzing the relationship between $\ln a a$ and $\ln R_{\mathrm{z}}$, this study shows that the $a a$ values are all in between the two lines of $a a_{\mathrm{t}}=e^{2.44} R_{\mathrm{Z}}^{2 / 7}$ and $a a_{\mathrm{b}}=e^{1.36} R_{\mathrm{Z}}^{2 / 7}$ defined solely by $R_{\mathrm{z}}$. According to the two lines, two ways can be selected to divide the $a a$ index into two components. If one is chosen as the baseline $a a_{\mathrm{b}}$ similar to the R component used by Feynman (1982), the remainder $a a_{\mathrm{u}}=a a-a a_{\mathrm{b}}$ (part of $a a$ above $a a_{\mathrm{b}}$ ) will have a null correlation with the former $(r=0.09)$, implying an independent decomposition. On the other hand, if one is chosen as the top-line $a a_{\mathrm{t}}$, the "minus remainder" $a a_{\mathrm{d}}=-\left(a a-a a_{\mathrm{t}}\right)$ will well follow the former $(r=0.78)$. The second decomposition is equivalent to dividing $a a_{\mathrm{t}}$ into two terms of $a a$ and $a a_{\mathrm{d}}$ (part of $a a_{\mathrm{t}}$ above $a a)$ with a null correlation $(r=0.00)$. With this decomposition, the $a a_{\mathrm{t}}$ term is interpreted as a nonlinear relation of $a a$ with $R_{\mathrm{Z}}$ and $a a_{\mathrm{d}}$ as a decay in transmission (due to energy loss). All the $a a$ indices can be mathematically expressed as $a a=e^{2.44 \pm 0.05-\eta} R_{\mathrm{Z}}^{2 / 7 \pm 0.02}$ for $0 \leq \eta \leq 1.08$. The decay index $\eta$ is mainly modulated by the solar wind speed $V(r=-0.67)$, and is almost independent of both the magnetic field $B$ of solar wind and the Dst index (ring current), as can be seen in Table 3 and Fig. 4.

The $a a_{\mathrm{b}}$ and $a a_{\mathrm{u}}$ terms in this study correspond similarly to the $\mathrm{R}$ and I components, respectively, with the main discrepancy that the $\mathrm{R}$ component is linearly related to $R_{\mathrm{Z}}$ (Eq. 2), while $a a_{\mathrm{b}}$ is related to $R_{\mathrm{z}}$ in the form of a power-law (Eq. 5). The $a a_{\mathrm{u}}$ or $a a_{\mathrm{I}}$ (part of $a a$ above $a a_{\mathrm{R}}$ ) component tends to have a $90^{\circ}\left(\sim 3\right.$-yr) rather than a $180^{\circ}$ phase shift relative to $a a_{\mathrm{b}}$ or $R_{\mathrm{Z}}$ (Fig. 3), so that the correlation coefficient between $a a_{\mathrm{b}}$ and $a a_{\mathrm{u}}$ is close to zero $(r=0.09)$.

To demonstrate the period in $a a_{\mathrm{u}}$, we obtain from Eqs. (5)(8), (15), and (18),

$$
\begin{aligned}
a a & =a a_{\mathrm{t}}-a a_{\mathrm{d}}(\eta)=e^{1.08-\eta} a a_{\mathrm{b}}, \\
a a_{\mathrm{u}}(\eta) & =a a-a a_{\mathrm{b}}=\left(e^{1.08-\eta}-1\right) a a_{\mathrm{b}} .
\end{aligned}
$$

Therefore, (i) the reason for the periodic variation in $a a_{\mathrm{u}}$ (or $\left.a a_{\mathrm{I}}\right)$ is only due to the periodic $a a_{\mathrm{b}}$ even if $\eta$ is not a constant. (ii) The reason for the $90^{\circ}$ phase shift of $a a_{\mathrm{u}}$ to $a a_{\mathrm{b}}$ is as follows. The recurrent geomagnetic activity is prevalent throughout the declining phase of the cycle (Wang and Sheeley, 2009), which may be related to the irregularity of the decay processes. The lag time of $a a$ to $R_{\mathrm{Z}}$ and the decay time contribute a phase $\phi$ to $a a_{\mathrm{u}}(\eta)$ such that $\eta=\eta^{\prime}+i \phi$, where $i$ is the imaginary unit. The phase $\phi$ has an average of about $\bar{\phi}=\pi / 2$ in the almost randomly distributed range of $[0, \pi]$. Thus, the correlation coefficient between $a a_{\mathrm{b}}$ and $a a_{\mathrm{u}}$ is close to zero (because the correlation coefficient between $\sin (t)$ and $\sin (t+\pi / 2)$ is zero).
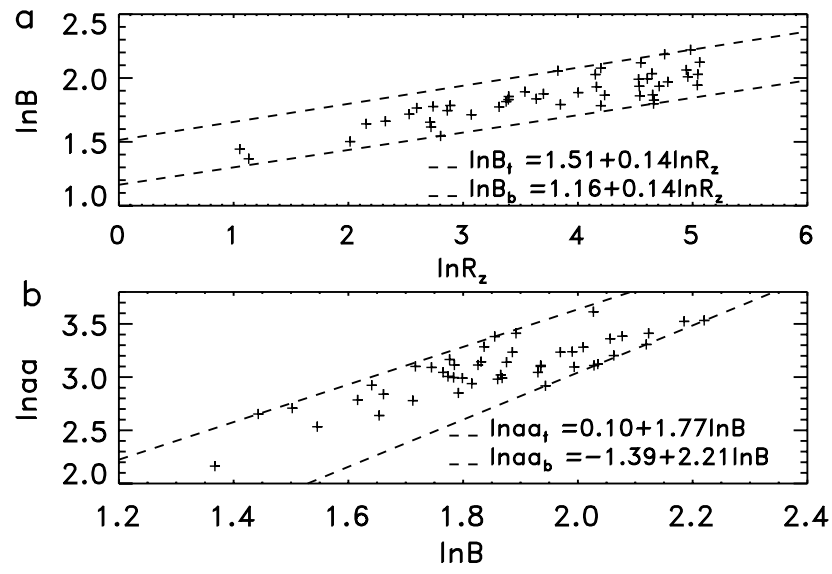

Fig. 5. (a) Scatter plot of $\ln B$ against $\ln R_{\mathrm{Z}}$ (pluses). The data points are all in between the two parallel (dashed) lines of $\ln B_{\mathrm{t}}$ (top) and $\ln B_{\mathrm{b}}$ (base). (b) Scatter plot of $\ln a a$ against $\ln B$ (pluses). The data points are all in between the two (dashed) lines of $\ln a a_{\mathrm{t}}$ (top) and $\ln a a_{\mathrm{b}}$ (base).

The fact that there are more decays at solar maxima than at solar minima is related to or can explain (partly) the following phenomena. (i) The "pearls" of $\sim 1$-year pulsations in $B_{\mathrm{Z}}$ are less near the maxima (Papitashvili et al., 2000). (ii) There is more mixing of fast and slow solar wind plasma at a solar maximum (Bame et al., 1976; Tu and Marsch, 1995; Richardson et al., 2000). (iii) The intensity of galactic cosmic rays (GCR) is reversely correlated with $R_{\mathrm{z}}$ (Nagashima et al., 1991; Stamper et al., 1999). (iv) The geomagnetic activity lags behind the solar activity for a longer time at a solar maximum than at a solar minimum (Legrand and Simon, 1981; Wang and Sheeley, 2009; Wilson, 1990; Du, 2011b,c). (v) Some activities may have stronger correlations with $R_{\mathrm{z}}$ around solar maxima than around solar minima.

It is well known that there are two main solar sources of geomagnetic activity (Legrand and Simon, 1981; Venkatesan et al., 1982; Feynman, 1982; Legrand and Simon, 1989a,b; Gonzalez et al., 1990; Venkatesan et al., 1991; Echer et al., 2004): one is related to the transient phenomena, and another is related to recurrent high-speed solar wind streams. Different activities have different properties before arriving at the magnetosphere and will undergo different interaction processes with the magnetosphere and ionosphere. For example, Fig. 5 shows the scatter plots of (a) $\ln B$ against $\ln R_{\mathrm{z}}$ and (b) $\ln a a$ against $\ln B$. One can see that the data points in Fig. 5a are all in between the two parallel (dashed) lines,

$$
\begin{aligned}
& \ln B_{\mathrm{t}}=1.51+0.14 \ln R_{\mathrm{z}}, \\
& \ln B_{\mathrm{b}}=1.16+0.14 \ln R_{\mathrm{z}} .
\end{aligned}
$$

The data points in Fig. $5 \mathrm{~b}$ are all in between the two (dashed) lines,

$$
\begin{aligned}
\ln a a_{\mathrm{t}} & =0.10+1.77 \ln B, \\
\ln a a_{\mathrm{b}} & =-1.39+2.21 \ln B .
\end{aligned}
$$



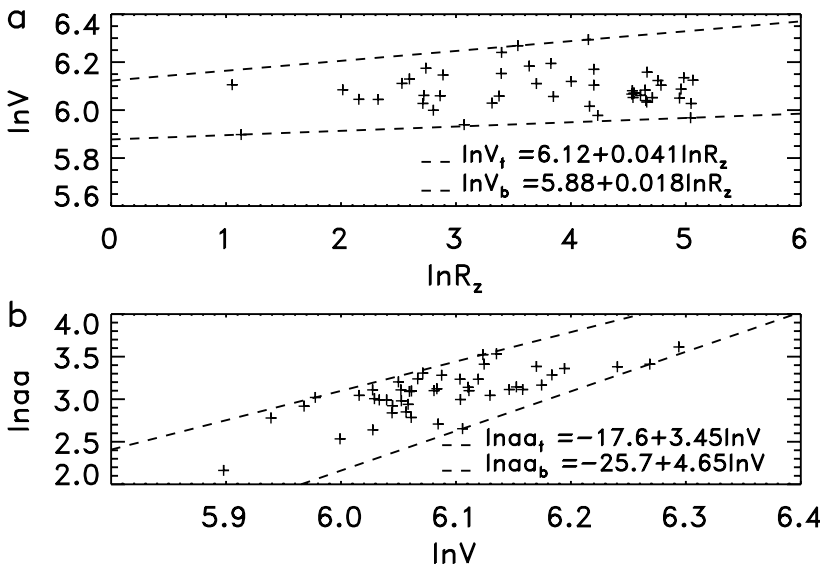

Fig. 6. (a) Scatter plot of $\ln V$ against $\ln R_{\mathrm{Z}}$ (pluses). The data points are all in between the two (dashed) lines of $\ln V_{\mathrm{t}}$ (top) and $\ln V_{\mathrm{b}}$ (base). (b) Scatter plot of $\ln a a$ against $\ln V$ (pluses). The data points are all in between the two (dashed) lines of $\ln a a_{\mathrm{t}}$ (top) and $\ln a a_{\mathrm{b}}$ (base).

As another example, Fig. 6 shows the scatter plots of (a) $\ln V$ against $\ln R_{\mathrm{Z}}$ and (b) $\ln a a$ against $\ln V$. One can also see that the data points in Fig. 6a are all in between the two (dashed) lines,

$$
\begin{aligned}
& \ln V_{\mathrm{t}}=6.12+0.041 \ln R_{\mathrm{z}}, \\
& \ln V_{\mathrm{b}}=5.88+0.018 \ln R_{\mathrm{z}} .
\end{aligned}
$$

The data points in Fig. $6 \mathrm{~b}$ are all in between the two (dashed) lines,

$$
\begin{aligned}
\ln a a_{\mathrm{t}} & =-17.6+3.45 \ln V, \\
\ln a a_{\mathrm{b}} & =-25.7+4.65 \ln V .
\end{aligned}
$$

Now we analyze the bivariate-fit of $\ln a a$ (solid) to both $\ln R_{\mathrm{Z}}$ (dashed) and $\ln V$ (dash-dotted) from 1964 to 2010, as shown in Fig. 7a. The dotted line indicates the fitted result $\left(\ln a a_{\mathrm{f}}\right)$, with the regression equation given by

$\ln a a=0.15 \ln R_{\mathrm{Z}}+2.35 \ln V-11.79$.

The correlation coefficient between $\ln a a$ and $\ln a a_{\mathrm{f}}(r=$ $0.91)$ is slightly higher than $(r=0.89)$ between $a a$ and the fitted result by the bivariate-fit of $a a$ to both $R_{\mathrm{Z}}$ and $V$.

Figure $7 \mathrm{~b}$ shows the scatter plot of $\ln a a$ against $\ln a a_{\mathrm{f}}$ (pluses). It is seen that the data points are all in between two (nearly parallel) lines,

$$
\begin{aligned}
\ln a a_{\mathrm{t}} & =0.17+1.01 \ln a a_{\mathrm{f}}, \\
\ln a a_{\mathrm{b}} & =0.08+0.90 \ln a a_{\mathrm{f}} .
\end{aligned}
$$

Therefore, the solar activity $\left(R_{\mathrm{Z}}\right)$ is related to the solar winds $(B, V)$ with values being in between two certain levels, and the solar winds $(B, V)$ are related to the geomagnetic activities $(a a)$ with values being in between another two certain levels. In turn, the solar activity $\left(R_{\mathrm{z}}\right)$ will be related to the geomagnetic activities $(a a)$ that have a lowest and a highest

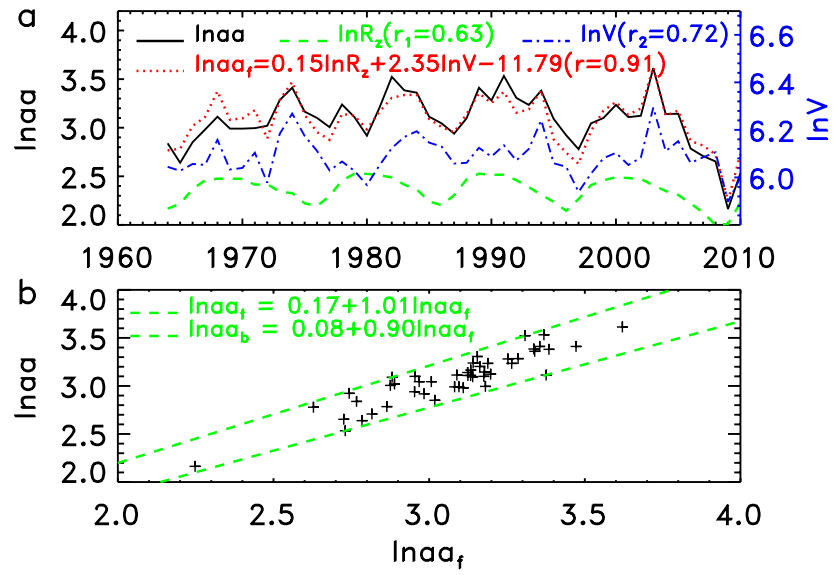

Fig. 7. (a) $\ln a a$ (solid), $\ln R_{\mathrm{Z}}$ (dashed), $\ln V$ (dash-dotted), and the fitted result $\ln a a_{\mathrm{f}}$ (dotted) by the bivariate of $\ln R_{\mathrm{Z}}$ and $\ln V$. (b) Scatter plot of $\ln a a$ against $\ln a a_{\mathrm{f}}$ (pluses). The data points are all in between the two (dashed) lines of $\ln a a_{\mathrm{t}}$ (top) and $\ln a a_{\mathrm{b}}$ (base).

level. Two ways can also be used to decompose an index $(B$, $V, a a)$ into two components according the lowest or highest level of activity, as was the case in the previous section.

The above results imply that the source activities $(B, V)$ of $a a$ play a role of mid-processes in the formation of $a a$ from solar magnetic field activity $\left(R_{\mathrm{Z}}\right)$. These processes are similar to the relationship between $a a$ and $R_{\mathrm{Z}}$ discussed in the previous section. The results in Figs. 5a and 6a indicate that the source activities $(B, V)$ have undergone decay processes before arriving at the magnetosphere (e.g., in the solar corona). The results in Figs. $5 \mathrm{~b}$ and $6 \mathrm{~b}$ indicate that the interactions of these activities with the magnetosphere and ionosphere will undergo further decays when generating $a a$. Generally speaking, the relationship between $a a$ and $R_{\mathrm{Z}}$ (Eq. 17) is the integrated effect of the above $R_{\mathrm{Z}}-B(V)-a a$ (and $R_{\mathrm{Z}}$-CME- $\left.a a\right)$ relationships. Thus, the decay term $\left(a a_{\mathrm{d}}\right)$ should include the decays of solar winds $(B, V)$, and the decay range (DR) may extend to the solar corona in this sense.

For ease of understanding, we discussed in Sect. 4.2 the decay process mainly in the magnetosphere and ionosphere. In Sect. 4.1, $R_{\mathrm{z}}$ is assumed to be associated with a certain amount of solar energy $\left(E_{\mathrm{t}}\right)$ potential of generating geomagnetic activity $(a a)$. This statement is consistent with the suggestion that sunspots are related to the energy supply to the corona (de Toma et al., 2000; Temmer et al., 2003). The energy $\left(E_{\mathrm{t}}\right)$ should have been related to $a a_{\mathrm{t}}$ (or a similar formula) if there were no energy loss $\left(E_{\mathrm{d}}\right)$ in the decay processes or if $E_{\mathrm{d}}$ were all used to generate $a a$ in the same way as the transient phenomena did.

However, it is not the case that all the solar energy $\left(E_{\mathrm{t}}\right)$ can generate or is directly related to geomagnetic activity $(a a)$. The energy transmission from solar surface to the magnetosphere and ionosphere may involve very complex processes. One process $\left(E_{\mathrm{b}}\right)$ is associated with the transient phenomena 
and follows the sunspot predominantly in a linear or nonlinear manner $\left(a a_{\mathrm{R}}\right.$ or $\left.a a_{\mathrm{b}}\right)$. Another process $\left(E_{\mathrm{t}}-E_{\mathrm{b}}\right)$ is associated with other (recurrent) phenomena such as solar winds. The energy $\left(E_{\mathrm{t}}-E_{\mathrm{b}}=E_{\mathrm{u}}+E_{\mathrm{d}}\right)$ for the latter process has undergone a loss $\left(E_{\mathrm{d} 1}\right.$, part of $\left.E_{\mathrm{d}}\right)$ before arriving at the magnetosphere and will undergo another loss $\left(E_{\mathrm{d} 2}=E_{\mathrm{d}}-E_{\mathrm{d} 1}\right)$ in the interactions with the magnetosphere and ionosphere. The energy loss $\left(E_{\mathrm{d}}\right)$ corresponds to the decay term $\left(a a_{\mathrm{d}}\right)$, which is related to solar (magnetic field) activity $\left(R_{\mathrm{Z}}\right)$ because the energy $\left(E_{\mathrm{t}}-E_{\mathrm{b}}\right)$ is associated with $R_{\mathrm{z}}$ as $E_{\mathrm{b}}$ is. It is shown in Sect. 4.2 that the decay $a a_{\mathrm{d}}$ precedes $a a$, which illustrates the fact that the generation of $a a$ occurs after the decay $\left(a a_{\mathrm{d}}\right)$ or the energy loss $\left(E_{\mathrm{d}}\right)$. The energy $E_{\mathrm{u}}$ is the remainder of $E_{\mathrm{t}}-E_{\mathrm{b}}=E_{\mathrm{u}}+E_{\mathrm{d}}$ after the (decay) loss $E_{\mathrm{d}}$. The geomagnetic activity $\left(a a_{\mathrm{u}}\right)$ generated by $E_{\mathrm{u}}$ is almost uncorrelated with $a a_{\mathrm{b}}$, which is due to the various processes in the formation of both $a a_{\mathrm{b}}$ and $a a_{\mathrm{u}}$.

The solar energy $\left(E_{\mathrm{t}}\right)$ can generate various solar activity phenomena such as solar flares, filament/prominence eruptions, energetic protons, CMEs, and solar winds (Gosling et al., 1976; Legrand and Simon, 1989a; Feminella and Storini, 1997; Sheeley et al., 1999; Forbes et al., 2006). These activities have lost part of their energies when passing over the solar chromospheric layer and corona. It is well known that most of these activities lag behind $R_{\mathrm{z}}$ from several months to a few years, which reflects the decay processes in the transmission. The energy loss $\left(E_{\mathrm{d} 1}\right)$ for the decay process of one activity may generate other activities, including various electric-magnetic radiations and the variations in density and temperature of the solar chromospheric layer and corona. Therefore, most solar activities are well correlated with $R_{\mathrm{Z}}$ (or $E_{\mathrm{t}}$ ), which is the reason for the good correlation of $a a_{\mathrm{d}}$ with $R_{\mathrm{Z}}$. The relationships between these activities and $R_{\mathrm{Z}}$ are similar to that between $a a$ and $R_{\mathrm{Z}}$ before arriving at the magnetosphere, as can be seen in Figs. 5a and 6a for two examples of the $B-R_{\mathrm{Z}}$ and $V-R_{\mathrm{z}}$ relationships, respectively.

Similarly, the interactions of the source activities with the magnetosphere and ionosphere can also generate a series of activity phenomena such as the ring current, auroral current, and the variations in density and temperature of the Earth's atmosphere. The energy loss $\left(E_{\mathrm{d} 2}\right)$ for the decay process of one activity may generate other activities, and not all these activities are completely related to $a a$. The relationships between $a a$ and these activities are similar to that between $a a$ and $R_{\mathrm{z}}$, as shown in Figs. $5 \mathrm{~b}$ and $6 \mathrm{~b}$ for two examples of the $a a-B$ and $a a-V$ relationships, respectively. In summary, $a a$ is the synthesis effect of all the above processes.

The main points of this paper may be summarized as follows:

1. The $a a$ index is in between the two levels of $a a_{\mathrm{t}}=$ $e^{2.44} R_{\mathrm{Z}}^{2 / 7}$ and $a a_{\mathrm{b}}=e^{1.36} R_{\mathrm{Z}}^{2 / 7}$.

2. aa can be decomposed into two independent components: $a a=a a_{\mathrm{b}}+a a_{\mathrm{u}}(r=0.09) . a a_{\mathrm{t}}$ can be divided into two independent terms: $a a_{\mathrm{t}}=a a+a a_{\mathrm{d}}(r=0.00)$.
3. All the $a a$ values can be expressed as $a a=a a_{\mathrm{t}}-a a_{\mathrm{d}}=$ $e^{2.44 \pm 0.05-\eta} R_{\mathrm{Z}}^{2 / 7 \pm 0.02}$ for $0 \leq \eta \leq 1.08$, where $\eta$ refers to the "decay index", modulated mainly by the solar wind speed.

Acknowledgements. The authors are grateful to the anonymous referees for suggestive comments, which greatly improved the present manuscript. This work is supported by the National Natural Science Foundation China through grants 10973020, 40890161, and 10921303, Chinese Academy of Sciences through grant YYYJ1110, and National Basic Research Program of China through grant 2011CB811406.

Topical Editor R. Nakamura thanks J. Lei and another anonymous referee for their help in evaluating this paper.

\section{References}

Bame, S. J., Asbridge, J. R., Feldman, W. C., and Gosling, J. T.: Solar cycle evolution of high-speed solar wind streams, Astrophys. J., 207, 977-980, 1976.

Crooker, N. U., Feynman, J., and Gosling, J. T.: On the high correlation between long-term averages of solar wind speed and geomagnetic activity, J. Geophys. Res., 82, 1933-1937, 1977.

Demetrescu, C. and Dobrica, V.: Signature of Hale and Gleissberg solar cycles in the geomagnetic activity, J. Geophys. Res., 113, A02103, doi:10.1029/2007JA012570, 2008.

de Toma, G., White, O. R., and Harvey, K. L.: A Picture of Solar Minimum and the Onset of Solar Cycle 23. I. Global Magnetic Field Evolution, Astrophys. J., 529, 1101-1114, 2000.

Du, Z. L.: The Relationship between Prediction Accuracy and Correlation Coefficient, Solar. Phys., 270, 407-416, 2011 a.

$\mathrm{Du}, \mathrm{Z}$. L.: The correlation between solar and geomagnetic activity - Part 2: Long-term trends, Ann. Geophys., Ann. Geophys., 29, 1341-1348, doi:10.5194/angeo-29-1341-2011, $2011 \mathrm{~b}$.

Du, Z. L.: The correlation between solar and geomagnetic activity - Part 3: An integral response model, Ann. Geophys., 29, 10051018, doi:10.5194/angeo-29-1005-2011, 2011c.

Du, Z. L., Li, R., and Wang, H. N.: The Predictive Power of Ohl's Precursor Method, Astron. J., 138, 1998-2001, 2009.

Echer, E., Gonzalez, W. D., Gonzalez, A. L. C., Gonzalez, W. D., Gonzalez, A. L. C., Prestes, A., Vieira, L. E. A., Dal Lago, A., Guarnieri, F. L., and Schuch, N. J.: Long-term correlation between solar and geomagnetic activity, J. Atmos. Sol. Terr. Phys., 66, 1019-1025, 2004.

Feminella, F. and Storini, M.: Large-scale dynamical phenomena during solar activity cycles, Astron. Astrophys., 322, 311-319, 1997.

Feynman, J.: Implications of solar cycles 19 and 20 geomagnetic activity for magnetospheric processes, Geophys. Res. Lett., 7, 971-973, 1980.

Feynman, J.: Geomagnetic and solar wind cycles, 1900-1975, J. Geophys. Res., 87, 6153-6162, 1982.

Feynman, J.: Reply, J. Geophys. Res., 88, 8139-8140, 1983.

Feynman, J. and Crooker, N. U.: The solar wind at the turn of the century, Nature, 275, 626-627, 1978.

Forbes, T. G., Linker, J. A., Chen, J., Cid, C., Kota, J., Lee, M. A., Mann, G., Mikic, Z., Potgieter, M. S., Schmidts, J. M., Siscoe, G. L., Vainio, R., Antiochos, S. K., and Riley, P.: CME Theory and Models, Space Sci. Rev., 123, 251-302, 2006. 
Garrett, H. B., Dessler, A. J., and Hill, T. W.: Influence of solar wind variability on geomagnetic activity, J. Geophys. Res., 79, 4603-4610, 1974.

Gonzalez, W. D., Gonzalez, A. I. C., and Tsurutani, B. T.: Dualpeak solar cycle distribution of intense geomagnetic storms, Planet. Space Sci., 38, 181-187, 1990.

Gosling, J. T., Hildner, E., MacQueen, R. M., Munro, R. H., Poland, A. I., and Ross, C. L.: The speeds of coronal mass ejection events, Solar Phys., 48, 389-397, 1976.

Hathaway, D. H. and Wilson, R. M.: Geomagnetic activity indicates large amplitude for sunspot cycle 24, Geophys. Res. Lett., 33, L18101, doi:10.1029/2006GL027053, 2006.

Johnson, J. R. and Wing, S.: A solar cycle dependence of nonlinearity in magnetospheric activity, J. Geophys. Res., 110, A04211, doi:10.1029/2004JA010638, 2005.

Leamon, R. J., Matthaeus, W. H., Smith, C. W., Zank, G. P., Mullan, D. J., and Oughton, S.: MHD-driven Kinetic Dissipation in the Solar Wind and Corona, Astrophys. J., 537, 1054-1062, 2000.

Legrand, J. P. and Simon, P. A.: Ten cycles of solar and geomagnetic activity, Solar Phys., 70, 173-195, 1981.

Legrand, J. P. and Simon, P. A.: Comment on 'geomagnetic and solar wind cycles, 1900-1975' by Joan Feynman, J. Geophys. Res., 88, 8137-8138, 1983.

Legrand, J. P. and Simon, P. A.: Solar cycle and geomagnetic activity: A review for geophysicists. I - The contributions to geomagnetic activity of shock waves and of the solar wind, Ann. Geophys., 7, 565-578, 1989a.

Legrand, J. P. and Simon, P. A.: Solar cycle and geomagnetic activity: A review for geophysicists. II - The solar sources of geomagnetic activity and their links with sunspot cycle activity, Ann. Geophys., 7, 579-593, 1989b.

Li, Y.: Predictions of the features for sunspot cycle 23, Sol. Phys., 170, 437-445, 1997.

Lukianova, R., Alekseev, G., and Mursula, K.: Effects of station relocation in the aa index, J. Geophys. Res., 114, A02105, doi:10.1029/2008JA013824, 2009.

Matthaeus, W. H., Weygand, J. M., Chuychai, P., Dasso, S., Smith, C. W., and Kivelson, M. G.: Interplanetary Magnetic Taylor Microscale and Implications for Plasma Dissipation, Astrophys. J. Lett., 678, L141-L144, 2008.

Mayaud, P. N.: The aa indices: A 100-year series characterizing the magnetic activity, J. Geophys. Res., 77, 6870-6874, 1972.

Nagashima, K., Fujimoto, K., and Tatsuoka, R.: Nature of solarcycle and heliomagnetic-polarity dependence of cosmic rays, inferred from their correlation with heliomagnetic spherical surface harmonics in the period 1976-1985, Planet. Space Sci., 39, 1617-1635, 1991.

Nevanlinna, H. and Kataja, E.: An extension of the geomagnetic activity index series aa for two solar cycles (1844-1868), Geophys. Res. Lett., 20, 2703-2706, 1993.

Papitashvili, V. O., Papitashvili, N. E., and King, J. H.: Solar cycle effects in planetary geomagnetic activity: Analysis of 36-year long OMNI dataset, Geophys. Res. Lett., 27, 2797-2800, 2000.
Richardson, I. G., Cliver, E. W., and Cane, H. V.: Sources of geomagnetic activity over the Solar cycle: relative importance of coronal mass ejections, high-speed streams, and slow Solar wind, J. Geophys. Res., 105, 18203-18213, 2000.

Russell, C. T. and McPherron, R. L.: Semiannual variation of geomagnetic activity, J. Geophys. Res., 78, 92-108, 1973.

Schatten, K. H., Scherrer, P. H., Svalgaard, L., and Wilcox, J. M.: Using dynamo theory to predict the sunspot number during solar cycle 21, Geophys. Res. Lett., 5, 411-414, 1978.

Sheeley, N. R., Walters, J. H., Wang, Y.-M., and Howard, R. A.: Continuous tracking of coronal outflows: Two kinds of coronal mass ejections, J. Geophys. Res., 104, 24739-24768, 1999.

Simon, P. A. and Legrand, J. P.: Solar cycle and geomagnetic activity: A review for geophysicists. II - The solar sources of geomagnetic activity and their links with sunspot cycle activity, Ann. Geophys., 7, 579-593, 1989.

Snyder, C. W., Neugebauer, M., and Rao, U. R.: The Solar Wind Velocity and Its Correlation with Cosmic-Ray Variations and with Solar and Geomagnetic Activity, J. Geophys. Res., 68, 63616370, 1963.

Stamper, R., Lockwood, M., Wild, M. N., and Clark, T. D. G.: Solar causes of the long-term increase in geomagnetic activity, J. Geophys. Res., 104, 28325-28342, 1999.

Svalgaard, L.: Geomagnetic activity: Dependence on solar wind parameters, in: Coronal Holes and High Speed Wind Streams, edited by: Zirker, J. B., Colorado Ass. U. Press, Boulder, p. 371, 1977.

Temmer, M., Veronig, A., and Hanslmeier, A.: Does solar flare activity lag behind sunspot activity? Sol. Phys., 215, 111-126, 2003.

Tsurutani, B. T., Gonzalez, W. D., Gonzalez, A. L. C., Guarnieri, F. L., Gopalswamy, N., Grande, M., Kamide, Y., Kasahara, Y., Lu, G., Mann, I., McPherron, R., Soraas, F., and Vasyliunas, V.: Corotating solar wind streams and recurrent geomagnetic activity: A review, J. Geophys. Res., 111, A07S01, doi:10.1029/2005JA011273, 2006.

Tu, C. Y. and Marsch, E.: MHD structures, waves and turbulence in the solar wind: observations and theories, Space Sci. Rev., 73, 1-210, 1995.

Venkatesan, D., Shulka, A. K., and Agrawal, S. P.: Cosmic ray intensity variations and two types of high speed soalr streams, Solar Phys., 81, 375-381, 1982.

Venkatesan, D., Ananth, A. G., Graumann, H., and Pillai, S.: Relationship between solar and geomagentic activity, J. Geophys. Res., 96, 9811-9813, 1991.

Wang, Y. M. and Sheeley, N. R.: Understanding the Geomagnetic Precursor of the Solar Cycle, Astrophys. J., 694, L11-L15, 2009.

Wilson, R. M.: On the level of skill in predicting maximum sunspot number - A comparative study of single variate and bivariate precursor techniques, Solar Phys., 125, 143-155, 1990. 